\title{
APPENDIX A: SUPPLEMENTARY MATERIAL
}

REMARK A.1 (Fourth-moment condition). Some of the technical results used in the proofs also require that the real i.i.d. random variables of Assumption (A2) in Section 2.1 have a finite fourth moment in addition to mean zero and unit variance. We apologize for not mentioning this additional requirement explicitly in Assumption (A2).

On the other hand, it appears that in practice a finite fourth moment is not needed for the nonlinear shrinkage methodology to still work; see Section 6.4.

Before proving Proposition 4.1, it is instructive to first state and prove a simpler result only claiming pointwise convergence of the estimated solutions. We will then see that this simpler proof can be extended relatively easily to also cover the more general claim of uniform convergence.

Proposition A.1. Let $\left\{\widehat{H}_{n}\right\}$ be a sequence of probability measures with $\widehat{H}_{n} \Rightarrow H$. Let $\left\{\widehat{c}_{n}\right\}$ be a sequence of positive real numbers with $\widehat{c}_{n} \rightarrow c$. Let $K \subseteq(0, \infty)$ be a compact interval satisfying $y_{x} \in K$. Let $\widehat{y}_{n, x} \equiv \min _{y \in K} g_{\widehat{H}_{n}, \widehat{c}_{n}}(y, x)$. It then holds that $\widehat{y}_{n, x} \rightarrow y_{x}$.

Proof. Assume $K=\left[k_{1}, k_{2}\right]$. Define $B \equiv\left\{x+i y: x \in\left[u_{1}, u_{2}\right], y \in K\right\}$, which implies $B \subseteq \mathbb{C}^{+}$.

We first claim that

$$
m_{L \widehat{H}_{n}}(z) \rightarrow m_{L H}(z) \quad \text { uniformly in } z \in B \text {. }
$$

Recalling that for any c.d.f. $G$, we have $m_{L G}(z)=1+z m_{G}(z)$ and by the compactness of the set $B$, this results will follow from

$$
m_{\widehat{H}_{n}}(z) \rightarrow m_{H}(z) \quad \text { uniformly in } z \in B
$$

which we establish now.

For fixed $z \in B$, consider the function

$$
h_{z}(\tau) \equiv \frac{\tau}{\tau-z} .
$$

Then it is easy to see that there exist two finite constants $d_{1}, d_{2}$, depending only on $k_{1}>0$ but not on $z$, such that

$$
\left|h_{z}\left(\tau_{1}\right)-h_{z}\left(\tau_{2}\right)\right| \leq d_{1}\left|\tau_{1}-\tau_{2}\right| \quad \text { and } \sup _{\tau}\left|h_{z}(\tau)\right| \leq d_{2}
$$

The fact that convergence in distribution of $\widehat{H}_{n}$ to $H$ is equivalent to convergence to zero of the bounded-Lipschitz metric between $\widehat{H}_{n}$ and $H$ then implies (A.2); for example, see Pollard (1984, Chapter IV, Example 22). In turn, we have thus established (A.1) as well. But (A.1) immediately implies

$$
g_{\widehat{H}_{n}, \widehat{c}_{n}}(y, x) \rightarrow g_{H, c}(y, x) \quad \text { uniformly in } y \in K
$$


We note the following two facts:

$$
\forall \varepsilon>0 \exists \delta>0 \text { such that } \inf _{y \in K,\left|y-y_{x}\right| \geq \varepsilon} g_{H, c}(y, x) \geq \delta
$$

and

$$
g_{\widehat{H}_{n}, \widehat{c}_{n}}\left(\widehat{y}_{n, x}, x\right)=o(1),
$$

where (A.6) follows from $g_{\widehat{H}_{n}, \widehat{c}_{n}}\left(\widehat{y}_{n, x}, x\right) \leq g_{\widehat{H}_{n}, \widehat{c}_{n}}\left(y_{x}, x\right),\left(\right.$ A.4), and $g_{H, c}\left(y_{x}, x\right)=0$.

By the triangular inequality,

$$
\begin{aligned}
g_{H, c}\left(\widehat{y}_{n, x}, x\right) & \leq\left|g_{H, c}\left(\widehat{y}_{n, x}\right)-g_{\widehat{H}_{n}, \widehat{c}_{n}}\left(\widehat{y}_{n, x}\right)\right|+\left|g_{\widehat{H}_{n}, \widehat{c}_{n}}\left(\widehat{y}_{n, x}\right)\right| \\
& =\left|g_{H, c}\left(\widehat{y}_{n, x}\right)-g_{\widehat{H}_{n}, \widehat{c}_{n}}\left(\widehat{y}_{n, x}\right)\right|+o(1) \quad \text { by }(\mathrm{A} .6) \\
& =o(1)+o(1) \quad \text { by }(\mathrm{A} .4) \\
& =o(1) .
\end{aligned}
$$

This last result together with (A.5) now implies $\widehat{y}_{n, x} \rightarrow y_{x}$.

Proof of Proposition 4.1. We start with part (i). Assume $K=\left[k_{1}, k_{2}\right]$. Define $B \equiv$ $\left\{x+i y: x \in\left[u_{1}, u_{2}\right], y \in K\right\}$, which implies $B \subseteq \mathbb{C}^{+}$.

By the same arguments leading up to (A.4) we can more generally establish that

$$
g_{\widehat{H}_{n}, \widehat{c}_{n}}(z) \rightarrow g_{H, c}(z) \quad \text { uniformly in } z \in B .
$$

We note the following two facts:

$$
\forall \varepsilon>0 \exists \delta>0 \text { such that } \inf _{x \in\left[u_{1}+\eta, u_{2}-\eta\right]}\left\{\inf _{y \in K,\left|y-y_{x}\right| \geq \varepsilon} g_{H, c}(y, x)\right\} \geq \delta
$$

and

$$
\sup _{x \in\left[u_{1}+\eta, u_{2}-\eta\right]} g_{\widehat{H}_{n}, \widehat{c}_{n}}\left(\widehat{y}_{n, x}, x\right)=o(1)
$$

where (A.9) follows from $g_{\widehat{H}_{n}, \widehat{c}_{n}}\left(\widehat{y}_{n, x}, x\right) \leq g_{\widehat{H}_{n}, \widehat{c}_{n}}\left(y_{x}, x\right),(\mathrm{A} .7)$, and $g_{H, c}\left(y_{x}, x\right)=0$.

To simplify the notation, let $I \equiv\left[u_{1}+\eta, u_{2}-\eta\right]$. By the triangular inequality,

$$
\begin{aligned}
\sup _{x \in I} g_{H, c}\left(\widehat{y}_{n, x}, x\right) & \leq \sup _{x \in I}\left|g_{H, c}\left(\widehat{y}_{n, x}\right)-g_{\widehat{H}_{n}, \widehat{c}_{n}}\left(\widehat{y}_{n, x}\right)\right|+\sup _{x \in I}\left|g_{\widehat{H}_{n}, \widehat{c}_{n}}\left(\widehat{y}_{n, x}\right)\right| \\
& =\sup _{x \in I}\left|g_{H, c}\left(\widehat{y}_{n, x}\right)-g_{\widehat{H}_{n}, \widehat{c}_{n}}\left(\widehat{y}_{n, x}\right)\right|+o(1) \quad \text { by (A.9) } \\
& =o(1)+o(1) \quad \text { by }(\mathrm{A} .7) \\
& =o(1) .
\end{aligned}
$$

This last result together with (A.9) now implies $\widehat{y}_{n, x} \rightarrow y_{x}$ uniformly in $x \in I=\left[u_{1}+\eta, u_{2}-\eta\right]$.

Part (ii) is proven analogously to part (i) by restricting attention to the set of probability one on which $\widehat{H}_{n} \Rightarrow H$ happens.

Proof of Proposition 4.2. The proof is similar to the proof of Proposition 4.1. The details are left to the reader.

imsart-aos ver. 2010/09/07 file: aos989.tex date: July 26, 2012 
Proof of Proposition 4.3. We start with part (i)(a). Fix $\lambda \in\left[\widetilde{z}_{1}+\widetilde{\delta}, \widetilde{z}_{2}-\widetilde{\delta}\right]$. Consider

$$
\left|\breve{m}_{F_{\widehat{H}_{n}, \widehat{c}_{n}}}(\lambda)-\breve{m}_{F}(\lambda)\right|=\left|\frac{1-\widehat{c}_{n}}{\widehat{c}_{n} \lambda}-\frac{1}{\widehat{c}_{n}} \frac{1}{\widehat{v}_{n, \lambda}}-\left(\frac{1-c}{c \lambda_{x}}-\frac{1}{c} \frac{1}{v_{\lambda}}\right)\right| .
$$

The function mapping $\lambda$ onto $v_{\lambda}$ is continuous, and therefore uniformly continuous, in $\lambda \in$ $\left[\widetilde{z}_{1}, \widetilde{z}_{2}\right]$. As $\lambda$ varies in $\left[\widetilde{z}_{1}+\widetilde{\delta}, \widetilde{z}_{2}-\widetilde{\delta}\right]$, the resulting $v_{\lambda}$ varies in a compact region in $\mathbb{C}^{+}$. Therefore, for any $\xi>0$, there exists $\kappa>0$ such that

$$
\left|\breve{m}_{F_{\widehat{H}_{n}}, \widehat{c}_{n}}(\lambda)-\breve{m}_{F}(\lambda)\right|<\xi \quad \text { as long as } \max \left\{\left|\widehat{c}_{n}-c\right|,\left|\widehat{v}_{n, \lambda}-v_{\lambda}\right|\right\}<\kappa .
$$

First, we can find $N_{1}$ such that $\left|\widehat{c}_{n}-c\right|<\kappa$ for all $n \geq N_{1}$. Second, by part (i) of Proposition 4.2, we can find $N_{2}$ such that $\left|\widehat{v}_{n, \lambda}-v_{\lambda}\right|<\kappa$ for all $n \geq N_{2}$, uniformly in $\lambda \in\left[\widetilde{z}_{1}+\widetilde{\delta}, \widetilde{z}_{2}-\widetilde{\delta}\right]$. Define $N \equiv \max \left\{N_{1}, N_{2}\right\}$. Then for all $n \geq N$, it holds that

$$
\left|\breve{m}_{\widehat{F}_{\widehat{H}_{n}, \widehat{c}_{n}}}(\lambda)-\breve{m}_{F}(\lambda)\right|<\xi \text {, uniformly in } \lambda \in\left[\widetilde{z}_{1}+\widetilde{\delta}, \widetilde{z}_{2}-\widetilde{\delta}\right] \text {. }
$$

Since $\xi$ can be chosen arbitrarily small, part (i)(a) obtains.

We now turn to part (i)(b). For any $\widetilde{\delta}>0$, it holds

$$
\begin{aligned}
\left\|\widehat{S}_{n}-S_{n}^{o r}\right\|^{2} & =\frac{1}{p} \sum_{i=1}^{p}\left(\frac{\lambda_{i}}{\left|1-\widehat{c}_{n}-\widehat{c}_{n} \lambda_{i} \breve{m}_{F_{\widehat{H}_{n}, \widehat{c}_{n}}}\left(\lambda_{i}\right)\right|^{2}}-\frac{\lambda_{i}}{\left|1-c-c \lambda_{i} \breve{m}_{F}\left(\lambda_{i}\right)\right|^{2}}\right)^{2} \\
& =\frac{1}{p} \sum_{\lambda_{i} \in\left[\tilde{z}_{1}+\widetilde{\delta}, \widetilde{z}_{2}-\widetilde{\delta}\right]}\left(\frac{\lambda_{i}}{\left|1-\widehat{c}_{n}-\widehat{c}_{n} \lambda_{i} \breve{m}_{F_{\widehat{H}_{n}, \widehat{c}_{n}}}\left(\lambda_{i}\right)\right|^{2}}-\frac{\lambda_{i}}{\left|1-c-c \lambda_{i} \breve{m}_{F}\left(\lambda_{i}\right)\right|^{2}}\right)^{2} \\
& +\frac{1}{p} \sum_{\lambda_{i} \notin\left[\tilde{z}_{1}+\widetilde{\delta}, \widetilde{z}_{2}-\widetilde{\delta}\right]}\left(\frac{\lambda_{i}}{\left|1-\widehat{c}_{n}-\widehat{c}_{n} \lambda_{i} \breve{m}_{F_{\widehat{H}_{n}, \widehat{c}_{n}}}\left(\lambda_{i}\right)\right|^{2}}-\frac{\lambda_{i}}{\left|1-c-c \lambda_{i} \breve{m}_{F}\left(\lambda_{i}\right)\right|^{2}}\right)^{2} \\
& \equiv A+B .
\end{aligned}
$$

By our general set of assumptions, in particular Assumption (A4), combined with the results of Bai and Silverstein (1998) and Mestre (2008, Section II), there exist two finite, non-zero constants $\kappa_{1}<\kappa_{2}$ such that $\kappa_{1} \leq \lambda_{i} \leq \kappa_{2}$ for all $i=1, \ldots, p$ and for all $n$ large enough.

Fix $\varepsilon>0$. First, we can pick $\widetilde{\delta}$ small enough to achieve $B \leq \varepsilon / 2$ eventually. To appreciate why, denote be $\mu(\widetilde{\delta})$ the mass that $F$ assigns to the set $\left[\widetilde{z}_{1}, \widetilde{z}_{1}+\widetilde{\delta}\right] \cup\left[\widetilde{z}_{2}-\widetilde{\delta}, \widetilde{z}_{2}\right]$, satisfying $\mu(\widetilde{\delta}) \rightarrow 0$ as $\widetilde{\delta} \rightarrow 0$. Then it is not too difficult to see that there exists a finite constant $\Delta$, possibly depending on $H$ and $c$, such that $B \leq \Delta \mu(\widetilde{\delta})$, for $n$ sufficiently large. The reason, in addition to $\kappa_{1} \leq \lambda_{i} \leq \kappa_{2}$, is that also the correction factors $\left|1-\widehat{c}_{n}-\widehat{c}_{n} \lambda_{i} \breve{m}_{F_{\widehat{H}_{n}, \widehat{c}_{n}}}\left(\lambda_{i}\right)\right|^{2}$ and $1 /\left|1-c-c \lambda_{i} \breve{m}_{F}\left(\lambda_{i}\right)\right|^{2}$ are bounded away from infinity. Then, choose $\widetilde{\delta}$ small enough so that $\mu(\widetilde{\delta}) \leq(2 / \varepsilon) \Delta$.

Having chosen and fixed $\widetilde{\delta}$, the first half of the assertion ensures that $A \leq \varepsilon / 2$ eventually. Again, we use here that $\kappa_{1} \leq \lambda_{i} \leq \kappa_{2}$ and that also also the correction factors $1 / \mid 1-c-$ $\left.c \lambda_{i} \breve{m}_{F}\left(\lambda_{i}\right)\right|^{2}$ are bounded away from infinity. This demonstrates part (i)(b).

Part(i)(c) can be handled in a very similar fashion.

Part (ii) is proven analogously to part (i) by focusing on the set of probability one on which $\widehat{H}_{n} \Rightarrow H$ happens. 
Before proving Theorem 5.1, we need to establish some auxiliary results.

Recall the following notation. For a grid $Q$ on the real line and for two c.d.f.s $G_{1}$ and $G_{2}$, define

$$
\left\|G_{1}-G_{2}\right\|_{Q} \equiv \sup _{t \in Q}\left|G_{1}(t)-G_{2}(t)\right|
$$

Lemma A.1. Let $\left\{G_{n}\right\}$ and $G$ be c.d.f.s on the real line, with the support of $G$ being compact. Let $\left\{Q_{n}\right\}$ be a sequence of grids on the real line, asymptotically covering the support of $G$, with grid sizes $\left\{\gamma_{n}\right\}$ satisfying $\gamma_{n} \rightarrow 0$. Further, assume that $\left\|G_{n}-G\right\|_{Q_{n}} \rightarrow 0$.

If $G$ is continuous, then $G_{n} \Rightarrow G$. In particular, $\sup _{t}\left|G_{n}(t)-G(t)\right| \rightarrow 0$.

Proof. Denote the compact support of $G$ by $[a, b]$. To prove the first part of the assertion, let $\varepsilon>0$. Fix $\delta>0$ such that for all $t<t^{\prime}$ with $t^{\prime}-t<\delta$, it holds $G\left(t^{\prime}\right)-G(t)<\varepsilon / 4$. Also fix $\phi>0$. First, there exists $N_{1}$ such that $\gamma_{n}<\delta$ for all $n \geq N_{1}$. Second, there exists $N_{2}$ such that $\sup _{t \in Q_{n}}\left|G_{n}(t)-G(t)\right|<\varepsilon / 4$ for all $n \geq N_{2}$. Third, there exists $N_{3}$ such that $Q_{n}$ covers $[a+\phi, b-\phi]$ for all $n \geq N_{3}$. Set $N \equiv \max \left\{N_{1}, N_{2}, N_{3}\right\}$. For an arbitrary $t \in[a+\phi, b-\phi]$ and for $n \geq N$, let $t_{n} \equiv \max \left\{\widetilde{t}: \widetilde{t} \in Q_{n}, \widetilde{t} \leq t\right\}$ and $t_{n}^{\prime} \equiv \min \left\{\widetilde{t}: \widetilde{t} \in Q_{n}, \widetilde{t} \geq t\right\}$, which implies $t_{n}-t_{n}^{\prime}<\delta$. Then, for all $n \geq N$,

$$
\begin{aligned}
\left|G_{n}(t)-G(t)\right| & \leq\left|G_{n}\left(t_{n}\right)-G\left(t_{n}^{\prime}\right)\right|+\left|G_{n}\left(t_{n}^{\prime}\right)-G\left(t_{n}\right)\right| \\
& \leq\left|G_{n}\left(t_{n}\right)-G\left(t_{n}\right)\right|+\left|G_{n}\left(t_{n}^{\prime}\right)-G\left(t_{n}^{\prime}\right)\right|+\frac{\varepsilon}{4}+\frac{\varepsilon}{4} \\
& \leq \frac{\varepsilon}{4}+\frac{\varepsilon}{4}+\frac{\varepsilon}{4}+\frac{\varepsilon}{4}=\varepsilon .
\end{aligned}
$$

Therefore, $G_{n}(t)$ converges to $G(t)$ for all $t \in[a+\phi, b-\phi]$; and since $\phi$ can be chosen arbitrarily, $G_{n}(t)$ converges to $G(t)$ for all $t \in(a, b)$. By picking $\phi$ sufficiently small such that $|G(a+\phi)| \leq \varepsilon$ and $|G(b-\varepsilon)| \geq 1-\varepsilon$, and by the monotonicity of c.d.f.s, it also follows that $\left|G_{n}(t)\right| \leq 2 \varepsilon$ for all $t \leq a$ as well as $\left|G_{n}(t)\right| \geq 1-2 \varepsilon$ for all $t \geq b$ as long as $n \geq N$ (where $N$ of course is allowed to depend on $\phi$.) Therefore, $G_{n}(t)$ converges to $G(t)$ for all $t$, which establishes $G_{n} \Rightarrow G$. The second part of the assertion follows immediately from the first part and Polya's Theorem.

LEMmA A.2. Let $G$ be a probability measure with compact support contained in $(0,+\infty)$ and let $d>0$. Let $\left\{\widehat{G}_{n}\right\}$ be a sequence of probability measures on the nonnegative real line with $\widehat{G}_{n} \Rightarrow G$ and let $\left\{\widehat{d}_{n}\right\}$ be a sequence of positive real numbers with $\widehat{d}_{n} \rightarrow d$. Also assume that there exists an interval $[a, b]$ contained in $(0,+\infty)$ such that $\operatorname{Supp}\left(\widehat{G}_{n}\right) \subseteq[a, b]$ for all $n$ large enough.

Then $F_{\widehat{G}_{n}, \widehat{d}_{n}} \Rightarrow F_{G, d}$.

Proof. Let $z_{j} \equiv i \cdot(1+1 / j)$, for $j=1,2, \ldots$ Then $\left\{z_{j}\right\}$ is an infinite sequence in $\mathbb{C}^{+}$with limit point $z_{0} \equiv i \in \mathbb{C}^{+}$. By Theorem 2 of Geronimo and Hill (2003), it is sufficient to show that, for all $z_{j}$,

$$
m_{F_{\widehat{G}_{n}, \widehat{d}_{n}}}\left(z_{j}\right) \rightarrow m_{F_{G, d}}\left(z_{j}\right)
$$

Recall the notation $m_{F_{\widetilde{H}, \widetilde{c}}}$ for the solution of the Marčenko-Pastur equation, for any probability measure $\widetilde{H}$ and for any $\widetilde{c}>0$. Namely, for each $z \in \mathbb{C}^{+}, m_{F_{\widetilde{H}, \widetilde{c}}}(z)$ is the unique solution 
for $m \in \mathbb{C}^{+}$to the equation

$$
m=\int_{-\infty}^{+\infty} \frac{1}{\tau[1-\widetilde{c}-\widetilde{c} z m]-z} d \widetilde{H}(\tau)
$$

Also, define the function

$$
\forall m, z \in \mathbb{C} \quad f_{\widetilde{H}, \widetilde{c}}(m, z) \equiv\left|m-\int_{-\infty}^{+\infty} \frac{1}{\tau[1-\widetilde{c}-\widetilde{c} z m]-z} d \widetilde{H}(\tau)\right|
$$

In this notation, for a given $z \in \mathbb{C}^{+}, m_{F_{\widetilde{H}, \widetilde{c}}}(z)$ is the unique solution for $m \in \mathbb{C}^{+}$to the equation $f_{\widetilde{H}, \widetilde{c}}(m, z)=0$. Alternatively, $m_{F_{\widetilde{H}, \widetilde{c}}}(z)$ is the unique minimizer over $m \in \mathbb{C}^{+}$of the function $f_{\widetilde{H}, \widetilde{c}}(\cdot, z)$. Note that the Stieltjes transform of any probability measure maps $\mathbb{C}^{+}$onto $\mathbb{C}^{+}$. So if $z \in \mathbb{C}^{+}$, then $m_{F_{\tilde{H}, \tilde{c}}}(z)$ is actually the unique minimizer over $m \in \mathbb{C}$ of the function $f_{\widetilde{H}, \tilde{c}}(\cdot, z)$.

Fix $z_{j}$ and use the following abbreviations: $\widehat{m}_{n, z_{j}} \equiv m_{F_{\widehat{G}_{n}, \widehat{d}_{n}}}\left(z_{j}\right)$ and $m_{z_{j}} \equiv m_{F_{G, d}}\left(z_{j}\right)$. The goal then is to show that $\widehat{m}_{n, z_{j}} \rightarrow m_{z_{j}}$.

We claim that there exists a compact set $S \subseteq \mathbb{C}$ such that $\widehat{m}_{n, z_{j}} \in S$ for all $n$. The proof is by means of contradiction. Assume the claim does not hold. Then there exists a subsequence $\left\{n_{k}\right\}$ such that $\left|\widehat{m}_{n_{k}, z_{j}}\right| \rightarrow \infty$. By the combined assumptions, we can then find $\Delta>0$ such that, for all $n_{k}$ large enough and for all $\tau \in[a, b]$,

$$
\frac{1}{\left|\tau\left[1-\widehat{d}_{n_{k}}-\widehat{d}_{n_{k}} z_{j} \widehat{m}_{n_{k}, z_{j}}\right]-z_{j}\right|} \leq \Delta
$$

implying that for all $n_{k}$ large enough,

$$
\begin{aligned}
\left|\widehat{m}_{n_{k}, z_{j}}\right| & =\left|\int_{-\infty}^{+\infty} \frac{1}{\tau\left[1-\widehat{d}_{n, k}-\widehat{d}_{n, k} z_{j} \widehat{m}_{n_{k}, z_{j}}\right]-z_{j}} d \widehat{G}_{n_{k}}(\tau)\right| \\
& =\left|\int_{a}^{b} \frac{1}{\tau\left[1-\widehat{d}_{n, k}-\widehat{d}_{n, k} z_{j} \widehat{m}_{n_{k}, z_{j}}\right]-z_{j}} d \widehat{G}_{n_{k}}(\tau)\right| \\
& \leq \int_{a}^{b} \frac{1}{\left|\tau\left[1-\widehat{d}_{n, k}-\widehat{d}_{n, k} z_{j} \widehat{m}_{n_{k}, z_{j}}\right]-z_{j}\right|} d \widehat{G}_{n_{k}}(\tau) \\
& \leq(b-a) \Delta .
\end{aligned}
$$

But this is in contradiction to $\left|\widehat{m}_{n_{k}, z_{j}}\right| \rightarrow \infty$. We may assume w.l.o.g. that $m_{z_{j}} \in S$ as well; otherwise sufficiently enlarge $S$.

We may further assume that $S$ is 'doubly nonnegative', that is, for all $m \in S$, it holds that $\operatorname{Re}(m) \geq 0$ as well as $\operatorname{Im}(m) \geq 0$. The reason is as follows. On the one hand, $\operatorname{Re}\left(\widehat{m}_{n, z_{j}}\right) \geq 0$ for all $n$ as well as $\operatorname{Re}\left(m_{z_{j}}\right) \geq 0$. For example, recalling that $\operatorname{Re}\left(z_{j}\right)=0$,

$$
\operatorname{Re}\left(m_{z_{j}}\right)=\operatorname{Re}\left(m_{F_{G, d}}\left(z_{j}\right)\right)=\int_{-\infty}^{\infty} \operatorname{Re}\left(\frac{1}{\lambda-z_{j}}\right) d F_{G, d}(\lambda)=\int_{-\infty}^{\infty} \frac{\lambda}{\left|\lambda-z_{j}\right|^{2}} d F_{G, d}(\lambda),
$$

where $F_{G, d}$ places all its mass on $[0,+\infty)$. On the other hand, since $z_{j} \in \mathbb{C}^{+}$, also $\operatorname{Im}\left(\widehat{m}_{n, z_{j}}\right)>0$ for all $n$ as well as $\operatorname{Im}\left(m_{z_{j}}\right)>0$.

We next claim that

$$
f_{\widehat{G}_{n}, d}\left(m, z_{j}\right) \rightarrow f_{G, d}\left(m, z_{j}\right) \quad \text { uniformly in } m \in S .
$$


To see why, for $m \in S$, consider the function

$$
h_{m, z_{j}}(\tau) \equiv \frac{1}{\tau\left[1-d-d z_{j} m\right]-z_{j}} .
$$

Since $S$ is compact, $\min \{\operatorname{Re}(m), \operatorname{Im}(m)\} \geq 0$ for all $m \in S, \operatorname{Re}\left(z_{j}\right)=0$, and $\operatorname{Im}\left(z_{j}\right) \geq 1$, there exist two finite constants $d_{1}$ and $d_{2}$, allowed to depend on $S$, such that

$$
\left|h_{m, z_{j}}\left(\tau_{1}\right)-h_{m, z_{j}}\left(\tau_{2}\right)\right| \leq d_{1}\left|\tau_{1}-\tau_{2}\right| \text { for } \tau_{1}, \tau_{2} \in[0,+\infty)
$$

and

$$
\sup _{\tau \in[0,+\infty)}\left|h_{m, z_{j}}(\tau)\right| \leq d_{2} .
$$

To see why, start with (A.13). It holds that

$$
\operatorname{Im}\left(\tau\left[1-d-d z_{j} m\right]-z_{j}\right)=-\left(\tau d\left[\operatorname{Re}\left(z_{j}\right) \operatorname{Im}(m)+\operatorname{Im}\left(z_{j}\right) \operatorname{Re}(m)\right]+\operatorname{Im}\left(z_{j}\right)\right) .
$$

Under the stated conditions, $\operatorname{Re}\left(z_{j}\right) \operatorname{Im}(m)+\operatorname{Im}\left(z_{j}\right) \operatorname{Re}(m) \geq 0$ and $\operatorname{Im}\left(z_{j}\right) \geq 1$. Therefore, as long as $\tau \geq 0$, it follows that

$$
\left|\tau\left[1-d-d z_{j} m\right]-z_{j}\right| \geq\left|\operatorname{Im}\left(\tau\left[1-d-d z_{j} m\right]-z_{j}\right)\right| \geq 1,
$$

implying that we may choose $d_{2} \equiv 1$.

Moving on to (A.12), let $\Delta \equiv \max _{m \in S}|m|$ and note that $\left|z_{j}\right| \leq 2$. Therefore, for any $\tau_{1}, \tau_{2} \in[0,+\infty)$,

$$
\begin{aligned}
\left|h_{m, z_{j}}\left(\tau_{1}\right)-h_{m, z_{j}}\left(\tau_{2}\right)\right| & =\left|\tau_{1}-\tau_{2}\right|\left|\frac{1-d-d z_{j} m}{\left(\tau_{1}\left[1-d-d z_{j} m\right]-z_{j}\right)\left(\tau_{2}\left[1-d-d z_{j} m\right]-z_{j}\right)}\right| \\
& =\left|\tau_{1}-\tau_{2}\right| \frac{\left|1-d-d z_{j} m\right|}{\left|\tau_{1}\left[1-d-d z_{j} m\right]-z_{j}\right|\left|\tau_{2}\left[1-d-d z_{j} m\right]-z_{j}\right|} \\
& =\left|\tau_{1}-\tau_{2}\right| \frac{\left|1-d-d z_{j} m\right|}{\left|\tau_{1}\left[1-d-d z_{j} m\right]-z_{j}\right|\left|\tau_{2}\left[1-d-d z_{j} m\right]-z_{j}\right|} \\
& \leq\left|\tau_{1}-\tau_{2}\right|(1+d+2 d \Delta),
\end{aligned}
$$

implying that we may choose $d_{1} \equiv(1+d+2 d \Delta)$.

Recall that convergence in distribution of $\widehat{G}_{n}$ to $G$ is equivalent to convergence to zero of the bounded-Lipschitz metric between $\widehat{G}_{n}$ and $G$; for example, see Pollard (1984, Chapter IV, Example 22). Furthermore, since $\widehat{G}_{n}$ and $G$ put all their mass on $[0, \infty)$, it is sufficient to start all integrals at $\tau=0$ rather than at $\tau=-\infty$. Therefore,

$$
\begin{aligned}
\int_{-\infty}^{+\infty} \frac{d \widehat{G}_{n}(\tau)}{\tau\left[1-d-d z_{j} m\right]-z_{j}} & =\int_{0}^{+\infty} \frac{1}{\tau\left[1-d-d z_{j} m\right]-z_{j}} d \widehat{G}_{n}(\tau) \\
& =\int_{0}^{\infty} h_{m, z_{j}}(\tau) d \widehat{G}_{n}(\tau) \\
& \rightarrow \int_{0}^{\infty} h_{m, z_{j}}(\tau) d G(\tau) \\
& =\int_{0}^{+\infty} \frac{1}{\tau\left[1-d-d z_{j} m\right]-z_{j}} d G(\tau) \\
& =\int_{-\infty}^{+\infty} \frac{1}{\tau\left[1-d-d z_{j} m\right]-z_{j}} d G(\tau) \quad \text { uniformly in } m \in S,
\end{aligned}
$$

imsart-aos ver. 2010/09/07 file: aos989.tex date: July 26, 2012 
which establishes (A.11). But (A.11), combined with the compactness of $S$, further implies that also

$$
f_{\widehat{G}_{n}, \widehat{d}_{n}}\left(m, z_{j}\right) \rightarrow f_{G, d}\left(m, z_{j}\right) \quad \text { uniformly in } m \in S .
$$

Summing up, we have the following facts: First, there exists a compact set $S \subseteq C$ such that $\widehat{m}_{n, z_{j}}$ is the unique minimizer of $f_{\widehat{G}_{n}, \widehat{d}_{n}}\left(\cdot, z_{j}\right)$ over $m \in S$ and $m_{z_{j}}$ is the unique minimizer of $f_{G, d}\left(\cdot, z_{j}\right)$ over $m \in S$. Second, the function $f_{G, d}\left(\cdot, z_{j}\right)$ is continuous in $m$. Third, the uniform convergence (A.14).

With these facts, $\widehat{m}_{n, z_{j}} \rightarrow m_{z_{j}}$ follows from arguments very similar to those used in the proof of Proposition A.1.

Proof of Theorem 5.1. We start with the proof of part (i). Since $c<1$, it follows from Silverstein and Choi (1995) that $F$ is continuously differentiable on all of $\mathbb{R}$. By Polya's Theorem it then follows that $\sup _{t}\left|F_{n}(t)-F(t)\right| \rightarrow 0$ a.s., implying that $\| F_{n}-F||_{Q_{n}} \rightarrow 0$ a.s. Also, by construction, $\left\|F_{\widehat{H}_{n}, \widehat{c}_{n}}-F_{n}\right\|_{Q_{n}} \leq\left\|F_{H, \widehat{c}_{n}}-F_{n}\right\|_{Q_{n}}$. Therefore,

$$
\begin{aligned}
\left\|F_{\widehat{H}_{n}, \widehat{c}_{n}}-F\right\|_{Q_{n}} & \leq\left\|F_{\widehat{H}_{n}, \widehat{c}_{n}}-F_{n}\right\|_{Q_{n}}+\left\|F_{n}-F\right\|_{Q_{n}} \\
& \leq\left\|F_{H, \widehat{c}_{n}}-F_{n}\right\|_{Q_{n}}+\left\|F_{n}-F\right\|_{Q_{n}} \\
& \leq\left\|F_{H, \widehat{c}_{n}}-F_{H, c}\right\|_{Q_{n}}+\left\|F_{H, c}-F_{n}\right\|_{Q_{n}}+\left\|F_{n}-F\right\|_{Q_{n}} \\
& =\left\|F_{H, \widehat{c}_{n}}-F\right\|_{Q_{n}}+2\left\|F_{n}-F\right\|_{Q_{n}} \rightarrow 0 \text { a.s. },
\end{aligned}
$$

where Lemma A.2 in conjunction with Polya's Theorem is used to show that $\left\|F_{H, \widehat{c}_{n}}-F\right\|_{Q_{n}} \rightarrow 0$. The desired result now follows from Lemma A.1.

We now turn to proving part (ii). By Theorem 2 of Geronimo and Hill (2003), it is sufficient to show that there exists an infinite sequence $\left\{v_{j}\right\}$ in $\mathbb{C}^{+}$with a limit point $v_{0} \in \mathbb{C}^{+}$such that

$$
m_{\widehat{H}_{n}}\left(v_{j}\right) \rightarrow m_{H}\left(v_{j}\right) \text { a.s. } \forall j \text {. }
$$

Recall the notation $m_{F_{\widetilde{H}, \widetilde{c}}}$ for the solution of the Marčenko-Pastur equation, for any probability measure $\widetilde{H}$ and for any $\widetilde{c}>0$. Namely, for each $z \in \mathbb{C}^{+}, m_{F_{\widetilde{H}, \widetilde{c}}}(z)$ is the unique solution for $m \in \mathbb{C}^{+}$to the equation

$$
m=\int_{-\infty}^{+\infty} \frac{1}{\tau[1-\widetilde{c}-\widetilde{c} z m]-z} d \widetilde{H}(\tau) .
$$

Analogously, to Subsection 2.2, also let

$$
\forall x \in \mathbb{R} \quad \underline{F}_{\widetilde{H}, \widetilde{c}}(x) \equiv(1-\widetilde{c}) \mathbb{1}_{[0,+\infty)}(x)+\widetilde{c} F_{\widetilde{H}, \widetilde{c}}(x)
$$

and

$$
\forall z \in \mathbb{C}^{+} \quad m_{\underline{F}_{\tilde{H}, \tilde{c}}}(z) \equiv \frac{\widetilde{c}-1}{z}+\widetilde{c} m_{F_{\widetilde{H}, \widetilde{c}}}(z) .
$$

Hence, for each $z \in \mathbb{C}^{+}, m_{\underline{F}_{\tilde{H}, \tilde{c}}}(z)$ is the unique solution for $m \in \mathbb{C}^{+}$to the equation

$$
m=-\left[z-\widetilde{c} \int_{-\infty}^{+\infty} \frac{\tau}{1+\tau m} d \widetilde{H}(\tau)\right]^{-1} .
$$


On $\mathbb{C}^{+}, m_{\underline{F}_{\tilde{H}, \tilde{c}}}(z)$ has a unique inverse, given by

$$
\forall m \in m_{\underline{F}_{\tilde{H}, \widetilde{c}}}\left(\mathbb{C}^{+}\right) \quad z_{\underline{F}_{\tilde{H}, \tilde{c}}}(m) \equiv-\frac{1}{m}+\widetilde{c} \int_{-\infty}^{+\infty} \frac{\tau}{1+\tau m} d \widetilde{H}(\tau) .
$$

Note that both $m_{\underline{F}_{\tilde{H}, \tilde{c}}}$ and $z_{\underline{F}_{\tilde{H}, \tilde{c}}}$ are continuous functions. Also in this notation, we have $\underline{F}=\underline{F}_{H, c}, m_{\underline{F}}=m_{\underline{F}_{H, c}}$, and $z_{\underline{F}}=z_{\underline{F}_{H, c}}$ then.

As Silverstein and Choi (1995) show,

$$
\forall m \in m_{\underline{F_{\tilde{H}}, \tilde{c}}}\left(\mathbb{C}^{+}\right) \quad z_{\underline{F}_{\tilde{H}, \tilde{c}}}(m)=-\frac{1}{m}+\frac{\widetilde{c}}{m}-\frac{\widetilde{c}}{m^{2}} m_{\widetilde{H}}\left(-\frac{1}{m}\right),
$$

which, letting $v \equiv-1 / m$, is equivalent to

$$
\text { (A.16) } \forall v \in \mathbb{C}^{+} \text {such that }-\frac{1}{v} \in m_{\underline{F}_{\widetilde{H}, \widetilde{c}}}\left(\mathbb{C}^{+}\right) \quad m_{\widetilde{H}}(v)=-\frac{1}{\widetilde{c} v^{2}}\left[z_{\underline{F}_{\widetilde{H}, \widetilde{c}}}\left(-\frac{1}{v}\right)-v+\widetilde{c} v\right] \text {. }
$$

For the special case of $\widetilde{H} \equiv H$ and $\widetilde{c} \equiv c$, this simplifies to

$$
\forall v \in \mathbb{C}^{+} \text {such that }-\frac{1}{v} \in m_{\underline{F}}\left(\mathbb{C}^{+}\right) \quad m_{H}(v)=-\frac{1}{c v^{2}}\left[z_{\underline{F}}\left(-\frac{1}{v}\right)-v+c v\right] .
$$

Let $M \subseteq \mathbb{C}^{+}$be a compact set contained in $m_{\underline{F}}\left(\mathbb{C}^{+}\right)$and also contained in $m_{\underline{F}_{\widehat{H}_{n}, \widehat{c}_{n}}}\left(\mathbb{C}^{+}\right)$, at least for $n$ large enough. Let $\left\{m_{j}\right\} \subseteq M$ be an infinite sequence with limit point $m_{0} \in M$. Let $v_{j} \equiv-1 / m_{j}$ and $v_{0} \equiv-1 / m_{0}$. Then $\left\{v_{j}\right\} \subseteq \mathbb{C}^{+}$with limit point $v_{0} \in \mathbb{C}^{+}$. Finally, let $z_{j} \equiv z_{\underline{F}}\left(m_{j}\right)$ and $z_{0} \equiv z_{\underline{F}}\left(m_{0}\right)$.

Part (i) of the theorem implies that $\underline{F}_{\widehat{H}_{n}, \widehat{c}_{n}} \Rightarrow \underline{F}$ a.s. It then follows from Corollary 1 of Geronimo and Hill (2003) that

$$
m_{\underline{F}_{\widehat{H}_{n}, \widehat{c}_{n}}}\left(z_{j}\right) \rightarrow m_{\underline{F}}\left(z_{j}\right) \text { a.s. } \forall j .
$$

In particular, the proof of Corollary 1 of Geronimo and Hill (2003) uses that convergence in distribution of probability measures implies convergence of integrals of bounded and continuous functions. A completely analogous argument can therefore be invoked to show that also

$$
z_{\underline{F}_{\widehat{H}_{n}, \widehat{c}_{n}}}\left(m_{j}\right) \rightarrow z_{\underline{F}}\left(m_{j}\right) \text { a.s. } \forall j
$$

or, equivalently, that

$$
z_{\underline{F}_{\widehat{H}_{n}, \widehat{c}_{n}}}\left(-\frac{1}{v_{j}}\right) \rightarrow z_{\underline{F}}\left(-\frac{1}{v_{j}}\right) \text { a.s. } \forall j .
$$

Using relation (A.16), with $\widetilde{H} \equiv \widehat{H}_{n}$ and $\widetilde{c} \equiv \widetilde{c}_{n}$, and relation (A.17), this implies that

$$
\begin{aligned}
m_{\widehat{H}_{n}}\left(v_{j}\right) & =-\frac{1}{\widehat{c}_{n} v_{j}^{2}}\left[z_{\underline{F}_{\widehat{H}_{n}, \widehat{c}_{n}}}\left(-\frac{1}{v_{j}}\right)-v_{j}+\widehat{c}_{n} v_{j}\right] \\
& \rightarrow-\frac{1}{c v_{j}^{2}}\left[z_{\underline{F}}\left(-\frac{1}{v_{j}}\right)-v_{j}+c v_{j}\right]=m_{H}\left(v_{j}\right) \text { a.s. } \forall j,
\end{aligned}
$$

which completes the proof of part (ii) the theorem.

Proof of Corollary 5.1. We start with the proof of part (i). Following El Karoui (2008), we call $H_{T_{n}}$ a discretization of $H$ on the grid $\left\{J_{n} / T_{n},\left(J_{n}+1\right) / T_{n}, \ldots, K_{n} / T_{n}\right\}$. For instance, 
we can choose $H_{T_{n}}$ to be a step function with $H_{T_{n}}(x) \equiv H(x)$ if $x=l / T_{n}, l \in \mathbb{N}$, and $H_{T_{n}}$ is constant on $\left[l / T_{n},(l+1) / T_{n}\right)$. If the support of $H$ is given by $\left[h_{1}, h_{2}\right]$, say, then the support of $H_{T_{n}}$ is contained in $\left[h_{1}-1 / T_{n}, h_{2}+1 / T_{n}\right]$. It is easy to see that for such a discretization $H_{T_{n}}$, it holds that $H_{T_{n}} \Rightarrow H$, as long as

$$
\exists b>0 \text { such that } \lambda_{p} \leq b \text { for all } n \text { sufficiently large and }
$$

(A.19) $\exists \gamma>0$ such that $J_{n} / T_{n} \leq h_{1}-\gamma$ and $K_{n} / T_{n} \geq h_{2}+\gamma$ for all $n$ sufficiently large .

First, (A.18) holds a.s. as shown by Bai and Silverstein (1998) and Mestre (2008, Section II), given our set of assumptions, in particular Assumption (A4). Second, the support of $F$ is denoted by $\left[\widetilde{z}_{1}, \widetilde{z}_{2}\right]$. On the one hand, it follows from Lemma 1.4 of Bai and Silverstein (1999) that $\widetilde{z}_{1}<h_{1}$ and $\widetilde{z}_{2}>h_{2}$. Therefore, it holds that $z_{1}=h_{1}-\delta_{1}$ and $z_{2}=h_{2}+\delta_{2}$ for some $\delta_{1}, \delta_{2}>0$. On the other hand, $F_{n} \Rightarrow F$ a.s., implying that $\lambda_{1} \leq \widetilde{z}_{1}+\delta_{1} / 2$ and $\lambda_{p} \geq \widetilde{z}_{2}-\delta_{2} / 2$ for $n$ sufficiently large a.s. So, letting $\gamma \equiv \min \left\{\delta_{1} / 2, \delta_{2} / 2\right\}$, condition (A.19) holds a.s. as well. Taken together, it follows that $H_{T_{n}} \Rightarrow H$ a.s.

By construction,

$$
\left\|F_{\widehat{H}_{n}, \widehat{c}_{n}}-F_{n}\right\|_{Q_{n}} \leq\left\|F_{H_{T_{n}}, \widehat{c}_{n}}-F_{n}\right\|_{Q_{n}} \leq\left\|F_{H_{T_{n}}, \widehat{c}_{n}}-F\right\|_{Q_{n}}+\left\|F-F_{n}\right\|_{Q_{n}} .
$$

We know that $\left\|F-F_{n}\right\|_{Q_{n}} \rightarrow 0$ a.s. So to establish part (i), it is sufficient to show that $\left\|F_{H_{T_{n}}, \widehat{c}_{n}}-F\right\|_{Q_{n}} \rightarrow 0$ a.s. Since $H_{T_{n}} \Rightarrow H$ a.s. and $\widehat{c}_{n} \rightarrow c$, it follows from Lemma A.2 and Polya's Theorem that $\sup _{t}\left|F_{H_{T_{n}}, \widehat{c}_{n}}(t)-F(t)\right| \rightarrow 0$ a.s., implying that $\left\|F_{H_{T_{n}}, \widehat{c}_{n}}-F\right\|_{Q_{n}} \rightarrow 0$ a.s.

But, having established part (i), part (ii) follows in exactly the same fashion as in the proof of Theorem 5.1.

Proof OF Corollary 5.2. We start with some preliminary results, leading up to the proof of part (ii). Let $G$ be a c.d.f. with continuous density $g$ and compact support $[a, b]$. For a grid $Q \equiv\left\{\ldots, t_{-1}, t_{0}, t_{1}, \ldots\right\}$ covering the support of $G$, the approximation to $G$ via trapezoidal integration is defined as in (5.3). Since $g$ is Lipschitz-continuous on $[a, b]$, there exists a (smallest) finite $\varepsilon>0$ such that $\left|g\left(t_{1}\right)-g\left(t_{2}\right)\right| \leq \varepsilon$ as long as $t_{1}-t_{2} \mid \leq \gamma$. Denote by $\widehat{g}_{Q}$ the density corresponding to $\widehat{G}_{Q}$. By definition of the trapezoidal rule, $\widehat{g}_{Q}$ is piecewise linear and agrees with $g$ at all points $t_{k} \in Q$. Since the grid size of $Q$ is given by $\gamma$, we may infer that

$$
\sup _{t}\left|g(t)-\widehat{g}_{Q}(t)\right| \leq 2 \varepsilon \text { and thus } \sup _{t}\left|G(t)-\widehat{G}_{Q}(t)\right| \leq 2 \varepsilon(b-a+2 \gamma) .
$$

We have assumed from the outset that $c<1$. By construction,

$$
\left\|\widehat{F}_{\widehat{H}_{n}, \widehat{c}_{n} ; Q_{n}}-F_{n}\right\|_{Q_{n}} \leq\left\|\widehat{F}_{H_{T_{n}}, \widehat{c}_{n} ; Q_{n}}-F_{n}\right\|_{Q_{n}} \leq\left\|\widehat{F}_{H_{T_{n}}, \widehat{c}_{n} ; Q_{n}}-F_{H_{T_{n}}, \widehat{c}_{n}}\right\|_{Q_{n}}+\left\|F_{H_{T_{n}}, \widehat{c}_{n}}-F_{n}\right\|_{Q_{n}} .
$$

It follows from the proof of Corollary 5.1 that $\left\|F_{H_{T_{n}}, \widehat{c}_{n}}-F_{n}\right\|_{Q_{n}} \rightarrow 0$ a.s. So if we can show that $\left\|\widehat{F}_{H_{T_{n}}, \widehat{c}_{n} ; Q_{n}}-F_{H_{T_{n}}, \widehat{c}_{n}}\right\|_{Q_{n}} \rightarrow 0$, it follows that $\left\|\widehat{F}_{\widehat{H}_{n}, \widehat{c}_{n} ; Q_{n}}-F_{n}\right\|_{Q_{n}} \rightarrow 0$ a.s.

For any probability measure $\widetilde{H}$, any $\widetilde{c}>0$, and any $\lambda \in(0,+\infty)$, let

$$
\breve{m}_{F_{\tilde{H}}, \widetilde{c}}(\lambda)=\lim _{z \in \mathbb{C}^{+} \rightarrow \lambda} m_{F_{\widetilde{H}, \widetilde{c}}}(z) \text {. }
$$


Also let $f_{\widetilde{H}, \widetilde{c}}(\lambda) \equiv \pi^{-1} \operatorname{Im}\left[\breve{m}_{F_{\widetilde{H}, \widetilde{c}}}(\lambda)\right]$ and define $f_{\widetilde{H}, \widetilde{c}}(0) \equiv 0$. Then

$$
\int_{-\infty}^{t} f_{\widetilde{H}, \widetilde{c}}(\lambda) d \lambda=\left\{\begin{array}{ll}
F_{\widetilde{H}, \widetilde{c}}(t) & \text { if } \widetilde{c}<1 \\
\widetilde{c} \underline{F}_{\widetilde{H}, \widetilde{c}}(t) & \text { if } \widetilde{c}>1
\end{array} .\right.
$$

We know that $f \equiv f_{H, c}$ is continuous, and therefore Lipschitz-continuous, on $\left[\widetilde{z}_{1}, \widetilde{z}_{2}\right]$ and constantly equal to zero outside $\left[\widetilde{z}_{1}, \widetilde{z}_{2}\right]$. Denote by $f_{\max }$ the maximum value of $f$. Since $H_{T_{n}} \Rightarrow H$, it follows from part (i) of Proposition 4.2 that, for every $\widetilde{\delta}>0$,

$$
f_{H_{T_{n}}, \widehat{c}_{n}}(\lambda) \rightarrow f(\lambda) \text { uniformly in } \lambda \in\left[\widetilde{z}_{1}+\widetilde{\delta}, \widetilde{z}_{2}-\widetilde{\delta}\right] .
$$

In particular, for every $\varepsilon>0$, we can find $N$ such that, for all $n \geq N$,

$$
\left|f_{H_{T_{n}}, \widehat{c}_{n}}(\lambda)-f(\lambda)\right|<\varepsilon \text { for all } \lambda \in\left[\widetilde{z}_{1}+\widetilde{\delta}, \widetilde{z}_{2}-\widetilde{\delta}\right] .
$$

For every $n$, the function $f_{H_{T_{n}}, \widehat{c}_{n}}$ is monotonically increasing near the left boundary of its support and monotonically decreasing near the right boundary of its support; see Silverstein and Choi (1995, Section 5). The compact support of $F$ is given by $\left[\widetilde{z}_{1}, \widetilde{z}_{2}\right]$. Lemma A.2 then implies that the support of $F_{H_{T_{n}}, \widehat{c}_{n}}$ is contained in $\left[\widetilde{z}_{1}-\eta_{n}, \widetilde{z}_{2}+\eta_{n}\right]$ for some positive sequence $\eta_{n} \rightarrow 0$, so

$$
f_{H_{T_{n}}, \widehat{c}_{n}}(\lambda)=0 \text { for } \lambda \notin\left[\widetilde{z}_{1}-\eta_{n}, \widetilde{z}_{2}+\eta_{n}\right] .
$$

And further, for $\eta_{n}$ and $\widetilde{\delta}$ sufficiently small and for $n$ sufficiently large, we may assume that

$$
f_{H_{T_{n}}, \widehat{c}_{n}}(\lambda) \leq 2 f_{\max } \quad \text { for all } \lambda \in\left[\widetilde{z}_{1}-\eta_{n}, \widetilde{z}_{1}+\widetilde{\gamma}_{n}\right] \cup\left[\widetilde{z}_{2}-\widetilde{\gamma}_{n}, \widetilde{z}_{2}+\eta_{n}\right] .
$$

Since $f$ is Lipschitz-continuous on $\left[\widetilde{z}_{1}, \widetilde{z}_{2}\right]$, for $\varepsilon>0$, there exists $\delta>0$ such that $\mid f\left(\lambda_{1}\right)-$ $f\left(\lambda_{2}\right) \mid \leq \varepsilon / 2$ for all $\lambda_{1}, \lambda_{2} \in\left[\widetilde{z}_{1}, \widetilde{z}_{2}\right]$ with $\left|\lambda_{1}-\lambda_{2}\right|<\delta$. From (A.21) it then follows that for $n$ large enough,

$$
\left|f_{H_{T_{n}}, \widehat{c}_{n}}\left(\lambda_{1}\right)-f_{H_{T_{n}}, \widehat{c}_{n}}\left(\lambda_{2}\right)\right| \leq \varepsilon \text { for all } \lambda_{1}, \lambda_{2} \in\left[\widetilde{z}_{1}+\widetilde{\delta}, \widetilde{z}_{2}-\widetilde{\delta}\right] \text { with }\left|\lambda_{1}-\lambda_{2}\right| \leq \delta .
$$

Applying the previous discussion for a general c.d.f. $G$ and a general grid $Q$ leading to (A.20) to the special cases of $F_{H_{T_{n}}, \widehat{c}_{n}}$ and $Q_{n}$, respectively, we thus obtain that, for $n$ large enough (in particular, satisfying $\gamma_{n} \leq \delta$ ),

$$
\sup _{\lambda \in\left[\widetilde{z} 1+\widetilde{\delta}, \widetilde{z}_{2}-\widetilde{\delta}\right]}\left|f_{H_{T_{n}}, \widehat{c}_{n}}(\lambda)-\widehat{f}_{H_{T_{n}}, \widehat{c}_{n} ; Q_{n}}(\lambda)\right| \leq 2 \varepsilon .
$$

Combining (A.22)-(A.24) yields, for $\epsilon$ and $\widetilde{\delta}$ small enough and for $n$ large enough,

$$
\sup _{\lambda \in \mathbb{R}}\left|F_{H_{T_{n}}, \widehat{c}_{n}}(\lambda)-\widehat{F}_{H_{T_{n}}, \widehat{c}_{n} ; Q_{n}}(\lambda)\right| \leq 2 \varepsilon\left(\widetilde{z}_{2}-\widetilde{z}_{1}+2 \delta\right)+4 f_{\max }\left(\eta_{n}+\widetilde{\delta}\right) .
$$

Since the right hand side of (A.25) can be made arbitrarily small, we have established that $\left\|\widehat{F}_{H_{T_{n}}, \widehat{c}_{n} ; Q_{n}}-F_{H_{T_{n}}, \widehat{c}_{n}}\right\|_{Q_{n}} \rightarrow 0$, which implies that $\left\|\widehat{F}_{\widehat{H}_{n}, \widehat{c}_{n} ; Q_{n}}-F_{n}\right\|_{Q_{n}} \rightarrow 0$ a.s., which in turn implies that

$$
\left\|\widehat{F}_{\widehat{H}_{n}, \widehat{c}_{n} ; Q_{n}}-F\right\|_{Q_{n}} \leq\left\|\widehat{F}_{\widehat{H}_{n}, \widehat{c}_{n} ; Q_{n}}-F_{n}\right\|_{Q_{n}}+\left\|F_{n}-F\right\|_{Q_{n}} \rightarrow 0 \text { a.s. } .
$$

Lemma A.1 then tells us that $\widehat{F}_{\widehat{H}_{n}, \widehat{c}_{n} ; Q_{n}} \Rightarrow F$ a.s. 
We now show that this implies part (ii) of the corollary, namely that $\widehat{H}_{n} \Rightarrow H$ a.s. by means of contradiction. To this end, assume that $\widehat{H}_{n} \Rightarrow H$ a.s. is not the case. The sequence $\left\{\widehat{H}_{n}\right\}$ is tight a.s. This is because the upper bound of the support of $H_{n}$ is given by $K_{n} / T_{n}$ which, by definition of $K_{n}$ satisfies $K_{n} / T_{n} \leq \lambda_{p}+1 / T_{n}$; and we know from Bai and Silverstein (1998) that for any $\varepsilon>0, \lambda_{p} \leq \widetilde{z}_{2}+\varepsilon$ for $n$ large enough a.s. Similar for the lower bound, or simply use zero as very crude lower bound. Therefore, if $\widehat{H}_{n} \Rightarrow H$ a.s. is not the case, there then exists a probability measure $H^{\prime} \neq H$ and a subsequence $\left\{n_{k}\right\}$ such that on a set with positive probability, we have $\widehat{H}_{n_{k}} \Rightarrow H^{\prime}$.

Similarly to an argument used in the proof of part (i) of Corollary 5.1 - with $\widehat{H}_{n_{k}}$ and $H^{\prime}$ now playing the roles of $H_{T_{n}}$ and $H$, respectively - it then follows that $\left\|F_{\widehat{H}_{n_{k}}, \widehat{c}_{n_{k}}}-F_{H^{\prime}, c}\right\|_{Q_{n_{k}}} \rightarrow 0$ on a set with positive probability. But it also holds that $\left\|\widehat{F}_{\widehat{H}_{n_{k}}, \widehat{c}_{n_{k}} ; Q_{n_{k}}}-F_{\widehat{H}_{n_{k}}, \widehat{c}_{n_{k}}}\right\|_{Q_{n_{k}}} \rightarrow 0$ similarly to an argument used above - with $F_{\widehat{H}_{n_{k}}, \widehat{c}_{n_{k}}}$ now playing the role of $F_{H_{T_{n}}, \widehat{c}_{n}}$. Together, we obtain that $\left\|\widehat{F}_{\widehat{H}_{n_{k}}, \widehat{c}_{n_{k}} ; Q_{n_{k}}}-F_{H^{\prime}, c}\right\|_{Q_{n_{k}}} \rightarrow 0$ on a set with positive probability. Since we are working under the assumption that $c<1$, both $F_{H}$ and $F_{H^{\prime}}$ are continuous. Lemma A.1 then tells us that $\sup _{t}\left|\widehat{F}_{\widehat{H}_{n_{k}} \widehat{c}_{n_{k}} ; Q_{n_{k}}}(t)-F_{H^{\prime}, c}(t)\right| \rightarrow 0$ on a set with positive probability. But this in contradiction to $\sup _{t}\left|\widehat{F}_{\widehat{H}_{n}, \widehat{c}_{n} ; Q_{n}}-F(t)\right| \rightarrow 0$ a.s. So the proof of part (ii) is accomplished.

We now can establish that $\left\|\widehat{F}_{\widehat{H}_{n}, \widehat{c}_{n} ; Q_{n}}-F_{\widehat{H}_{n}, \widehat{c}_{n}}\right\|_{Q_{n}} \rightarrow 0$ a.s., knowing that $\widehat{H}_{n} \Rightarrow H$ a.s., very much in the same way as we established before that $\left\|\widehat{F}_{H_{T_{n}}, \widehat{c}_{n} ; Q_{n}}-F_{H_{T_{n}}, \widehat{c}_{n}}\right\|_{Q_{n}}$, knowing that $H_{T_{n}} \Rightarrow H$. As a result, we obtain that $\left\|F_{\widehat{H}_{n}, \widehat{c}_{n}}-F\right\|_{Q_{n}} \rightarrow 0$ a.s. Invoking Lemma A.1 establishes part (i) then.

Parts (iii)-(iv) follow immediately from parts (i)-(ii) and Proposition 4.3, part (ii).

\section{ADDITIONAL REFERENCES}

BAi, Z. D. and Silverstein, J. W. (1999) Exact separation of eigenvalues of large-dimensional sample covariance matrices. Ann. Prob. 27 1536-1555.

Geronimo, J. S. and Hill, T. P. (2003). Necessary and sufficient condition that the limit of Stieltjes transforms is a Stieltjes transform. J. Approx. Theory 121 54-60.

Mestre, X. (2008). On the asymptotic behavior of the sample estimates of eigenvalues and eigenvectors of covariance matrices. IEEE Trans. Signal Process. 56 5353-5368.

Pollard, D. (1984) Convergence of Stochastic Processes. Springer Series in Statistics. Springer, New York. 